\title{
Examining the need for routine intensive care admission after surgical repair of nonsyndromic craniosynostosis: a preliminary analysis
}

\author{
Christopher M. Bonfield, MD, ${ }^{1,2}$ Jade Basem, ${ }^{2}$ D. Douglas Cochrane, MD, FRCSC, ${ }^{3}$ \\ Ash Singhal, MSc, MD, FRCSC, ${ }^{3}$ and Paul Steinbok, MBBS, FRCSC ${ }^{3}$ \\ 1Department of Neurological Surgery; and 'Surgical Outcomes Center for Kids, Vanderbilt University, Nashville, Tennessee; \\ and 'Division of Pediatric Neurosurgery, Department of Surgery, University of British Columbia and BC Children's Hospital, \\ Vancouver, British Columbia, Canada
}

OBJECTIVE At British Columbia Children's Hospital (BCCH), pediatric patients with nonsyndromic craniosynostosis are admitted directly to a standard surgical ward after craniosynostosis surgery. This study's purpose was to investigate the safety of direct ward admission and to examine the rate at which patients were transferred to the intensive care unit (ICU), the cause for the transfer, and any patient characteristics that indicate higher risk for ICU care.

METHODS The authors retrospectively reviewed medical records of pediatric patients who underwent single-suture or nonsyndromic craniosynostosis repair from 2011 to 2016 at BCCH. Destination of admission from the operating room (i.e., ward or ICU) and transfer to the ICU from the ward were evaluated. Patient characteristics and operative factors were recorded and analyzed.

RESULTS One hundred fourteen patients underwent surgery for single-suture or nonsyndromic craniosynostosis. Eighty surgeries were open procedures (cranial vault reconstruction, frontoorbital advancement, extended-strip craniectomy) and 34 were minimally invasive endoscope-assisted craniectomy (EAC). Sutures affected were sagittal in 66 cases ( 32 open, $34 \mathrm{EAC}$ ), coronal in 20 (15 unilateral, 5 bilateral), metopic in 23 , and multisuture in 5 . Only 5 patients who underwent open procedures $(6 \%)$ were initially admitted to the ICU from the operating room; the reasons for direct admission were as follows: the suggestion of preoperative elevated intracranial pressure, pain control, older-age patients with large reconstruction sites, or a significant medical comorbidity. Overall, of the 107 patients admitted directly to the ward (75 who underwent an open surgery, 32 who underwent an EAC), none required ICU transfer.

CONCLUSIONS Overall, the findings of this study suggest that patients with nonsyndromic craniosynostosis can be managed safely on the ward and do not require postoperative ICU admission. This could potentially increase cost savings and ICU resource utilization.

https://thejns.org/doi/abs/10.3171/2018.6.PEDS18136

KEYWORDS craniosynostosis; craniofacial surgery; pediatric; intensive care unit

A DMISSION of pediatric neurosurgical patients postoperatively to an intensive care unit (ICU), rather than a ward, for complex procedures is a common practice. However, many institutions are beginning to question the necessity of this. Admission to the ICU can be extremely costly, and the practice utilizes many resources that may potentially not be necessary for these pediatric patients in the absence of severe complications. The increasing safety of pediatric neurosurgical procedures and the more standardized protocols of care could lead to ward admission being adequate to handle any potential postoperative complications.

Cost analysis of ICU versus ward care has demonstrated a substantial difference and has brought attention to the

ABBREVIATIONS BCCH = British Columbia Children's Hospital; EAC = endoscope-assisted craniectomy; ICP = intracranial pressure; ICU = intensive care unit. SUBMITTED March 1, 2018. ACCEPTED June 22, 2018.

INCLUDE WHEN CITING Published online September 21, 2018; DOI: 10.3171/2018.6.PEDS18136. 
traditional standard of care of ICU admission even when the procedures have been uncomplicated. ${ }^{9}$ Research in this area is beginning to be completed for many types of neurosurgical procedures in cases of uncomplicated surgeries, mainly in adult populations. ${ }^{1-4,6,7,12}$ These studies have found that admission to a postoperative ward can be sufficient for the care of patients after many different neurosurgical procedures, such as elective craniotomy and for monitoring and care after traumatic brain injury. In these studies, there has been the suggestion that ward admission should be based on a risk-priority protocol and the importance of comorbidities as a determining factor for a higher level of care.

Pediatric neurosurgical patients, specifically those undergoing craniosynostosis repair, have not been studied as thoroughly on this topic of ward versus ICU admission. Just as many of the adult studies showed that comorbidities and intraoperative complications were indicators for ICU admission postoperatively, these factors have also been identified in initial studies in pediatric patients undergoing craniofacial surgery. ${ }^{5}$ Significant postoperative events were unusual, and it has been suggested that, after craniofacial surgery, most patients could safely be admitted to the ward for their care. One study of frontoorbital craniosynostosis repair in infants found that comorbidities significantly influenced a patient's success in the ward setting and directly compared the cost of postoperative ward admission with that of ICU admission..$^{10}$

In many institutions, children undergoing surgery for craniosynostosis repair are admitted to the ICU for immediate postoperative care. However, at British Columbia Children's Hospital (BCCH), pediatric patients treated for single-suture or nonsyndromic craniosynostosis are routinely admitted postoperatively directly to a standard surgical ward. The present study investigated the safety of admitting these patients directly to the ward and examined the rate at which patients were subsequently transferred to a higher level of care (i.e., ICU), the events that led to the transfer, and any characteristics of the patient that might place the patient at higher risk for needing ICU care.

\section{Methods}

After institutional review board approval, we reviewed the cases of pediatric patients $(<18$ years of age) who underwent surgery for craniosynostosis between 2011 and 2016. In all patients, nonsyndromic craniosynostosis repair was performed at $\mathrm{BCCH}$. Information collected included date of birth, date of surgery, age at surgery, hemoglobin levels (preoperatively, intraoperatively, and postoperatively), affected suture, if unilateral or bilateral surgery was performed, if a redo operation was needed, or if blood transfusion was given; we also examined any operative notes that indicated possible complications or relevant details. Patient information was collected from admission until discharge. We also assessed destination of admission from the operating room and whether transfer to the ICU from the ward was necessary.

Our general postoperative protocol is observation in the recovery room for 90-120 minutes before admitting patients to the ward. At $\mathrm{BCCH}$, the ward nurse-to-patient
TABLE 1. Patient characteristics and postoperative destination

\begin{tabular}{ccc}
\hline & \multicolumn{2}{c}{ No. of Patients } \\
\cline { 2 - 3 } Suture Affected & Overall & Admitted to the Ward Postop \\
\hline Sagittal & 66 & 63 \\
\hline Open & 32 & 31 \\
\hline EAC & 34 & 32 \\
\hline Coronal & 20 & 20 \\
\hline Unilateral & 15 & 15 \\
\hline Bilateral & 5 & 5 \\
\hline Metopic & 23 & 21 \\
\hline Multisuture & 5 & 3 \\
\hline Total & 114 & $107^{*}$ \\
\hline Open & 80 & 75 \\
\hline EAC & 34 & 32 \\
\hline
\end{tabular}

* Of the 107 patients admitted directly to the ward postoperatively, none required transfer to the ICU.

ratio is 3:1. Neurological and vital sign checks are every hour for the first 4 hours on admission, then once every 2 hours for 8 hours, and then once every 4 hours until discharge. Pain control involves a continuous morphine infusion protocol that has been described in previous publications. ${ }^{8,11}$

\section{Results}

Overall, 114 pediatric patients were included within this study for nonsyndromic craniosynostosis from 2011 to 2016 at BCCH. Eighty were open procedures (cranial vault reconstruction $[\mathrm{n}=6$ ], frontoorbital advancement [n $=43]$, extended-strip craniectomy $[n=31]$ ) and 34 were minimally invasive endoscope-assisted craniectomy (EAC). Affected sutures for each surgery were sagittal (n $=32$ open surgery, $\mathrm{n}=34 \mathrm{EAC})$, coronal $(\mathrm{n}=15$ unilateral, $\mathrm{n}=5$ bilateral), metopic (23), and multisuture (5). The average age of patients was 1.02 years (SD 1.02 years, range 0.18-5.88 years) for open procedures, and blood transfusion was performed in 12 patients (15\%) during open procedures. Two of the patients requiring transfusion were admitted directly to the ICU for other reasons.

As detailed in Table 1, 107 patients were admitted directly to the ward postoperatively (75 after open and 32 after EAC procedures). Overall, 5 of 80 patients $(6 \%)$ who underwent an open procedure were admitted to the ICU postoperatively. None of the patients admitted directly to the ward were transferred to the ICU during their postoperative stay (Table 2). Reasons for direct ICU admission were as follows: the suggestion of preoperative elevated intracranial pressure (ICP), the need for pain control, older patients with large reconstruction sites, or significant medical comorbidity. No patients had delayed-onset complications that required admission to the ICU.

\section{Discussion}

In the present 5-year retrospective review, no patient who underwent single-suture or nonsyndromic craniosynostosis repair admitted to the ward was thereafter trans- 
TABLE 2. Open surgery-treated patients postoperatively admitted to ICU

\begin{tabular}{ccccl}
\hline Pt No. & Age (mos) & Suture Affected & Procedure Type & \multicolumn{1}{c}{ Reason for Postop Admission to ICU From OR } \\
\hline 1 & 3 & Multisuture & Open & Suggested elevated ICP (scalloped bone, Chiari malformation) \\
\hline 2 & 26 & Multisuture & Open & Older patient, suggested elevated ICP (papilledema) \\
\hline 3 & 12 & Metopic & Open & Medical comorbidities (renal disease, laryngomalacia) \\
\hline 5 & 11 & Metopic & Open & Required sedation for pain control \\
\hline 5 & 71 & Sagittal & Open & Older patient, large reconstruction \\
\hline
\end{tabular}

$\mathrm{OR}=$ operating room; $\mathrm{Pt}=$ patient.

Only 5 of 80 open surgery-treated patients were admitted to the ICU from the operating room. Reasons for direct ICU admission were as follows: a suggestion of elevated preoperative ICP, pain control, older-age patients with large reconstruction, or a medical comorbidity.

ferred to the ICU. Only 6.14\% (7/114) were admitted directly to the ICU, and the reasons for admission were the suggestion of preoperative increased ICP, lack of a ward bed, older-age patients with large reconstruction sites, or a significant medical comorbidity. This percentage suggests that ward care is adequate for the majority of these postoperative patients. The leading factor for ICU determination was comorbidities, and in this study there were no patient characteristics that predicted transfer from the ward to the ICU, as no patients admitted to the ward ever required such a transfer.

Seruya et al. found that among patients who required ICU treatment, which was only $4.7 \%$ of their 107 patient cohort, there was a significantly higher incidence of comorbidities, intraoperative blood loss, and postoperative blood transfusion..$^{10}$ For patients admitted directly to the ICU in our study, comorbidities were the only factor we found. This also agrees with Goobie and colleagues in their risk-priority analysis protocol. For their study, 64\% of their 225-patient cohort were found to be, what they classified as, a low-risk group that could have been adequate for ward admission. ${ }^{5}$

The comparison of ward admission within a specialty unit as opposed to a general postoperative unit has not yet been studied but could bring to light more ways to update protocols and standard postoperative care in order to optimize high-care units, specifically for craniosynostosis repairs. While costs were not explicitly analyzed in the present study, ICU admission is known to utilize more resources and to be more costly to the patient and the hospital. Changing the standard of care such that such postoperative cases are routinely admitted directly to the ward rather than ICU could optimize ICU usage and reduce costs. A larger cohort than ours and more defined protocol could also be useful in determining specific risk factors for the safety of placing patients in the ward postoperatively after craniosynostosis repair.

It is also imperative to have a ward that is able to care for patients with such acuity and protocols have been established for postoperative care. Institutions without an ability to complete frequent neurological and vital sign checks may not have the capacity for these postoperative patients to be admitted to the ward.

The limitations of the present study are that it was a single-center institutional retrospective review of patients treated during a limited time period. This greatly reduced the available sample size and limits the generalizability to other institutions. Patients who had confirmed syndromic craniosynostosis were also excluded, which limited the overall craniofacial population available. Also, retrospective review is limited by medical record information and changing protocols. To appropriately assess the rate of transfer to the ICU after admission to a ward, given a power of 0.80 , we would need 2020 patients. Given the time frame and number of patients evaluated in this study (5 years, 114 patients), we would need to either evaluate practice patterns over a 90 -year period or conduct a multiinstitutional study with 18 sites contributing 115 patients each. A large multicenter study would be beneficial to further investigate this topic, and additional prospectively collected data (such as pain scores, family satisfaction, and costs) would be beneficial in future studies.

\section{Conclusions}

Overall, this study suggests that patients with nonsyndromic craniosynostosis can be managed safely on the ward and do not require routine admission to the ICU after surgery. This could potentially result in significant cost savings and more efficient ICU resource utilization. Further large-scale multicenter studies should be performed to investigate this finding.

\section{References}

1. Bee TK, Magnotti LJ, Croce MA, Maish GO, Minard G, Schroeppel TJ, et al: Necessity of repeat head CT and ICU monitoring in patients with minimal brain injury. J Trauma 66:1015-1018, 2009

2. Bui JQ, Mendis RL, van Gelder JM, Sheridan MM, Wright $\mathrm{KM}$, Jaeger M: Is postoperative intensive care unit admission a prerequisite for elective craniotomy? J Neurosurg 115:1236-1241, 2011

3. Gabel BC, Martin J, Crawford JR, Levy M: Questioning the need for ICU level of care in pediatric patients following elective uncomplicated craniotomy for brain tumors. J Neurosurg Pediatr 17:564-568, 2016

4. Godden DR, Patel M, Baldwin A, Woodwards RT: Need for intensive care after operations for head and neck cancer surgery. Br J Oral Maxillofac Surg 37:502-505, 1999

5. Goobie SM, Zurakowski D, Proctor MR, Meara JG, Meier PM, Young VJ, et al: Predictors of clinically significant postoperative events after open craniosynostosis surgery. Anesthesiology 122:1021-1032, 2015

6. Hecht N, Spies C, Vajkoczy P: Routine intensive care unitlevel care after elective craniotomy: time to rethink. World Neurosurg 81:66-68, 2014 
7. Mirza FA, Wang C, Pittman T: Can patients safely be admitted to a ward after craniotomy for resection of intra-axial brain tumors? Br J Neurosurg 32:201-205, 2018

8. Ou CH, Kent SK, Hammond AM, Bowen-Roberts T, Steinbok P, Warren DT: Morphine infusions after pediatric cranial surgery: a retrospective analysis of safety and efficacy. Can J Neurosci Nurs 30:21-30, 2008

9. Pastores SM, Dakwar J, Halpern NA: Costs of critical care medicine. Crit Care Clin 28:1-10, v, 2012

10. Seruya M, Sauerhammer TM, Basci D, Rogers GF, Boyajian MJ, Myseros JS, et al: Analysis of routine intensive care unit admission following fronto-orbital advancement for craniosynostosis. Plast Reconstr Surg 131:582e-588e, 2013

11. Warren DT, Bowen-Roberts T, Ou C, Purdy R, Steinbok P: Safety and efficacy of continuous morphine infusions following pediatric cranial surgery in a surgical ward setting. Childs Nerv Syst 26:1535-1541, 2010

12. Washington CW, Grubb RL Jr: Are routine repeat imaging and intensive care unit admission necessary in mild traumatic brain injury? J Neurosurg 116:549-557, 2012

\section{Disclosures}

The authors report no conflict of interest concerning the materi- als or methods used in this study or the findings specified in this paper.

\section{Author Contributions}

Conception and design: Bonfield, Steinbok. Acquisition of data: Bonfield, Basem, Steinbok. Analysis and interpretation of data: Bonfield, Basem, Cochrane, Singhal. Drafting the article: Bonfield, Basem. Critically revising the article: all authors. Reviewed submitted version of manuscript: all authors. Approved the final version of the manuscript on behalf of all authors: Bonfield. Study supervision: Steinbok.

\section{Supplemental Information}

\section{Previous Presentations}

Portions of this work were presented in abstract form at the Annual Meeting of the International Society for Pediatric Neurosurgery, Denver, Colorado, October 2018.

\section{Correspondence}

Christopher M. Bonfield: Vanderbilt University Medical Center, Nashville,TN. chris.bonfield@vanderbilt.edu. 